OPEN ACCESS

Edited by:

Attilio Marino,

Italian Institute of Technology, Italy

Reviewed by:

Ana Magalhães,

University of Porto, Portugal

Joseph T. Y. Lau,

University at Buffalo, United States

*Correspondence:

Michael O'Dwyer

michael.odwyer@nuigalway.ie

Specialty section:

This article was submitted to

Nanobiotechnology,

a section of the journal

Frontiers in Bioengineering and

Biotechnology

Received: 26 June 2019

Accepted: 17 September 2019

Published: 04 October 2019

Citation:

Natoni A, Bohara R, Pandit A and

O'Dwyer M (2019) Targeted

Approaches to Inhibit Sialylation of Multiple Myeloma in the Bone Marrow

Microenvironment.

Front. Bioeng. Biotechnol. 7:252. doi: 10.3389/fbioe.2019.00252

\section{Targeted Approaches to Inhibit Sialylation of Multiple Myeloma in the Bone Marrow Microenvironment}

\author{
Alessandro Natoni ${ }^{1}$, Raghvendra Bohara ${ }^{2}$, Abhay Pandit ${ }^{2}$ and Michael O'Dwyer ${ }^{1 *}$ \\ ${ }^{1}$ Apoptosis Research Centre, School of Medicine, National University of Ireland, Galway, Ireland, ${ }^{2}$ Centre for Research in \\ Medical Devices (CÚRAM), National University of Ireland, Galway, Ireland
}

Aberrant glycosylation modulates different aspects of tumor biology, and it has long been recognized as a hallmark of cancer. Among the different forms of glycosylation, sialylation, the addition of sialic acid to underlying oligosaccharides, is often dysregulated in cancer. Increased expression of sialylated glycans has been observed in many types of cancer, including multiple myeloma, and often correlates with aggressive metastatic behavior. Myeloma, a cancer of plasma cells, develops in the bone marrow, and colonizes multiple sites of the skeleton including the skull. In myeloma, the bone marrow represents an essential niche where the malignant cells are nurtured by the microenvironment and protected from chemotherapy. Here, we discuss the role of hypersialylation in the metastatic process focusing on multiple myeloma. In particular, we examine how increased sialylation modulates homing of malignant plasma cells into the bone marrow by regulating the activity of molecules important in bone marrow cellular trafficking including selectins and integrins. We also propose that inhibiting sialylation may represent a new therapeutic strategy to overcome bone marrow-mediated chemotherapy resistance and describe different targeted approaches to specifically deliver sialylation inhibitors to the bone marrow microenvironment.

\section{Keywords: multiple myeloma, microenvironment, sialylation, targeted delivery, chemotherapy, ST3GAL6,} E-selectin, integrins

\section{INTRODUCTION}

The development and progression of human malignancies from the first appearance of benign lesions to the emergence of deadly metastatic clones consist of a multi-step process involving the progressive acquisition of selective alterations that ultimately result in the pathological behavior of cancer cells. Glycosylation represents one of these processes that are often dysregulated in cancer. It is one of the most important co- and post-translational modifications that contributes to and modulates the many diverse functions of glycoconjugates. This extraordinary range of biological functions originates from the complexity and the diversity found within the glycan structure such as carbohydrate composition and linkage, anomeric state, branching, presence of additional modification (sulfation), and linkage to their aglycone part (carrier). As the majority of known glycoconjugates are exposed to the outer surface of the plasma membrane or secreted into the extracellular milieu, they mediate important biological processes such as cell adhesion, migration, immune response, as well as receptor activation and intracellular signaling. During tumor development, the glycan profile undergoes a profound and dynamic alteration that allows 
cancer cells to acquire novel traits necessary for tumor progression. These glycosylation changes are not random and involve, among others, de novo synthesis of new carbohydrate structures, premature termination of pre-existing glycans, and increased expression of terminal sialylated glycans. Acquisition of sialylated structures represents one of the most important modifications of the glycome during tumor development, and it is often associated with an aggressive metastatic phenotype. However, the study of the role of sialylation in cancer is still in its infancy and strategies to efficiently and safely target this important biological process are still lacking.

\section{MULTIPLE MYELOMA: A METASTATIC DISEASE THAT DEPENDS ON THE BONE MARROW MICROENVIRONMENT}

Multiple myeloma (MM) arises from clonal expansion of terminally differentiated plasma cells in the bone marrow (BM). $\mathrm{MM}$ is usually preceded by asymptomatic precursor states called Monoclonal Gammopathy of Undetermined Significance (MGUS) and Smoldering MM (SMM). Genetic abnormalities, epigenetic alterations, and microenvironmental factors cooperate in the development of symptomatic MM (Bianchi and Munshi, 2015). The BM microenvironment represents the perfect niche where MM cells proliferate and become resistant to chemotherapeutic drugs (Manier et al., 2012). A combination of soluble growth factors and adhesion molecules mediate these pro-survival and proliferative signaling pathways (Di Marzo et al., 2016). This extreme dependency on the BM suggests that malignant cells could be particularly vulnerable in the circulation where the effective concentration of a chemotherapeutic drug is higher than in the BM and where they are more susceptible to an immune response. Thus, MM cells must have evolved strategies to enhance their survival in the bloodstream such as mechanisms of immune evasion and efficient homing into the BM. Supporting this hypothesis is the evidence that MM is highly metastatic, colonizing different sites of the axial skeleton including the skull (Moschetta et al., 2017). Homing of MM cells into the $\mathrm{BM}$ is primarily mediated by stromal cell-derived factor $1 \alpha(\mathrm{SDF} 1 \alpha)$ and its receptor C-X-C chemokine receptor type 4 (CXCR4) (Alsayed et al., 2007). This chemokine also plays a role in adhesion and possibly retention of MM in the BM via $\alpha 4 \beta 1$ dependent adhesion on fibronectin and vascular cell adhesion molecule 1 (VCAM-1) (Gazitt and Akay, 2004; Parmo-Cabanas et al., 2004; Menu et al., 2006). Besides SDF1 $\alpha$, other molecules have been shown to be important in homing and adhesion of MM to the BM. These include integrin $\alpha 4 \beta 1, \alpha 4 / \beta 7$, and P-selectin glycoprotein ligand-1 (PSGL-1), all of which are highly expressed on MM cells (Sanz-Rodriguez et al., 1999; Florena et al., 2005; Neri et al., 2011). Notably, these molecules, including SDF1 $\alpha$, are also involved in cell adhesion-mediated drug resistance (CAMDR) and therefore represent attractive targets for MM therapy (Damiano et al., 1999; Azab et al., 2009; Muz et al., 2015; Waldschmidt et al., 2017). Although these molecules have been shown to be important in regulating critical biological processes involved in the progression and development of MM, little is known about how post-translational modifications influence their functions. Above all, the role of sialylation in regulating some of the biological functions of these molecules has only been recently recognized. Secretion of extracellular vesicles (EVs) by malignant plasma cells represents another important mechanism of MM dissemination (Colombo et al., 2019). Indeed, MM-EVs have been found both in MM patients' peripheral blood (PB) and $\mathrm{BM}$, and their levels in bloodstream positively correlate with the number of bone lesions (Zhang L. et al., 2019). It has been proposed that EVs have an important role in different steps of the metastatic process (Colombo et al., 2019). Due to their pro-coagulant activity, EVs could lead to platelet activation and polymerization of fibrinogen to fibrin, which in turn would enhance MM dissemination by protecting the malignant plasma cells in the circulation, favoring their seeding to distant sites and pre-conditioning the metastatic niche with platelet-derived cytokines (Labelle et al., 2014; Remiker and Palumbo, 2018; Nielsen et al., 2019). It has also been shown that MM-EVs contribute to neo-angiogenesis by inducing endothelial cell proliferation and formation of new blood vessels (Liu et al., 2014; Wang et al., 2016; Li et al., 2019; Zarfati et al., 2019). Whether MM-EV sialylation status is important for some or all their biological functions is yet to be confirmed. It is also tempting to speculate that MM-EVs could also alter the sialylation status of the metastatic niche favoring MM seeding and homing into the BM. Although this is just a hypothesis, it represents an exciting field for future studies.

\section{SIALYLATION: AN IMPORTANT CO- AND POST-TRANSLATIONAL MODIFICATION IN CANCER BIOLOGY}

Sialic acid refers to a group of $\sim 50$ chemical variants of the nine carbon sugar neuraminic acid that are strategically placed at the tip of glycans. At physiological $\mathrm{pH}$, sialic acids confer a negative charge to the underlying glycoproteins and glycolipids, which contribute to their physiological and biophysical functions. Indeed, sialic acids provide charge repulsion on human erythrocytes and other cells, preventing unwanted interactions of cells in the circulation. In the kidneys, sialic acids are critical in maintaining their normal filtering function (Gelberg et al., 1996), and extended polysialic acid chains can affect neuronal plasticity (Rutishauser, 2008). Moreover, sialic acids represent critical determinants of selectin ligands, which contribute to extravasation of immune cells to target organs and sites (Mcever, 2015). Pathologically, sialic acids are involved in many infectious diseases, being the targets for the binding of a large number of pathogenic organisms and their toxins, and in cancer (Varki, 2008). Indeed, aberrant sialylation is observed in many cancers and it seems to be a major contributor of the metastatic phenotype (Schultz et al., 2012).

Sialic acids can be added to the underlying sugars through different linkages: an $\alpha 2-3$ - or an $\alpha 2-6$-bond to galactose (Gal); an $\alpha 2-6$-bond to N-acetylgalactosamine (GalNAc) or Nacetylglucosamine (GlcNAc); and an $\alpha 2$-8-bond to another sialic acid, forming polysialic acid. These linkages are elaborated by a 
family of 20 sialyltransferases (STs), a class of glycosyltransferases that share the cytosine monophosphate (CMP)-sialic acid as substrate donor, but differ in tissue distribution, in the catalysis of the specific glycosidic linkage and in the recognition of the acceptor oligosaccharide and/or glycoconjugates (HarduinLepers et al., 2001). All the STs are highly stereoselective and catalyze the formation of an $\alpha$-linked sialic acid to a precise hydroxyl group on a specific saccharide residue. The combination of the acceptor saccharide residue and the precise hydroxyl group on this residue can be used to divide the family into four distinct sub-families. For example, ST6Gal enzymes catalyze the transfer of sialic acid to the 6'-hydroxyl group of a Gal residue, ST3Gal enzymes catalyze the transfer of sialic acid to the $3^{\prime}$ hydroxyl group of a Gal residue, ST3GalNAc enzymes catalyze the transfer of sialic acid to the $6^{\prime}$-hydroxyl group of a GalNac, and ST3Sia enzymes catalyze the transfer of sialic acid to the 8 -hydroxyl group of another sialic acid residue. The number of the STs exceeds by far the number of existing sialyl linkages, suggesting a certain degree of redundancy. However, although there are six STs capable of catalyzing the transfer of sialic acid to an underlying Gal residue in an $\alpha 2-3$ linkage, only two of them, namely, ST3GAL4 and ST3GAL6, have been implicated in synthetizing selectin ligands (Rodrigues and Macauley, 2018). Thus, it might be possible that the structure of the underlying glycans together with the protein or lipid backbone dictate the specificity of the different STs.

Aberrant sialylation can arise as a result of different mechanisms including overexpression of STs, dysfunction of the enzymes that controls the synthesis and availability of CPMsialic acids and downregulation of sialidase, the enzymes that cleave off sialic acids from glycans (Rodrigues and Macauley, 2018). Aberrant sialylation seems to be particularly important in promoting tumor dissemination, survival in the circulation, adhesion on the target endothelium, and extravasation (Schultz et al., 2012). For example, ST6GAL1 has been implicated in many metastatic tumors (Garnham et al., 2019). Physiologically, ST6GAL1 is involved in the generation of CD22 ligands that control B cell functions (Clark and Giltiay, 2018). However, upregulation of ST6GAL1 in cancer leads to enhanced migration and invasiveness by specifically inducing sialylation of the integrin $\beta 1$ (Seales et al., 2005; Christie et al., 2008; Shaikh et al., 2008). Selectin ligands represent another class of molecules that are regulated by sialylation and are instrumental in directing tumor cell adhesion on the endothelium of target organs during metastatic dissemination (Natoni et al., 2016). Sialyl Lewis (sLe) structures are essential determinants for the function of selectin ligands. These are tetrasaccharide structures composed of a GlcNAc-Gal backbone, an $\alpha 2-3$-linked sialic acid to Gal, and a fucose linked to GlcNAc either with an $\alpha 1-3\left(\operatorname{sLe}^{\mathrm{x}}\right)$ or an $\alpha 1-$ 4 linkage $\left(s L e^{\mathrm{a}}\right)$. Through selectin ligand/selectin interactions, tumor cells are able to tether and roll on the vascular endothelium in a process that closely mirror leukocyte extravasation during inflammation or homing (Gout et al., 2008). This represents the first step in cancer seeding and colonization of target organs. Notably, the expression of selectin ligands on cancer cells seems to correlate with metastatic phenotype (Fukuoka et al., 1998; Tatsumi et al., 1998; Ben-David et al., 2008; Geng et al., 2012; Li et al., 2013) and disease progression (Chien et al., 2013; Gakhar et al., 2013) and negatively correlate with patient survival (Amado et al., 1998; Baldus et al., 1998; Grabowski et al., 2000; Woodman et al., 2016).

Aberrant sialylation can indirectly promote metastatic spread by increasing survival of metastatic cells in the circulation. P-selectin ligands on tumor cells interact with P-selectin on platelets, inducing the formation of a platelet cloak that efficiently suppresses the innate immune system and promotes metastasis (Placke et al., 2012; Cluxton et al., 2019). Sialoglycans are also recognized by a class of immunomodulatory receptors named sialic acid-binding immunoglobulin-type lectins (Siglecs), which mainly serve as negative modulators of the immune system (Rodrigues and Macauley, 2018). In this respect, sialoglycans can be considered as self-associated molecular patterns (SAMPs) that can lead to inhibition of immune responses against self. Hypersialylation can also induce immune evasion by interference with the complement system via a "molecular cloaking" mechanism mediated by Factor $\mathrm{H}$ sequestration and dampening of the complement-mediated cell lysis and opsonization (Fedarko et al., 2000). Thus, hypersialylation can contribute to the metastatic phenotype of cancer cells with a variety of mechanisms that interfere with different biological processes spanning from immune surveillance to adhesion.

\section{ROLE OF SIALYLATION IN THE BIOLOGY OF MYELOMA}

The first evidence of altered sialylation in MM dates back to 1984, when an increase in ST activity was reported in mononuclear cells and serum derived from the $\mathrm{PB}$ and $\mathrm{BM}$ of MM patients compared to MGUS and healthy individuals (Cohen et al., 1989). However, despite this important observation, there was no functional characterization of this enhanced ST activity. Direct evidence of a role of aberrant sialylation in MM came from the observation that ST3GAL6, a ST responsible for the generation of a2-3-linked sialic acids, was highly expressed in MM and correlated with inferior overall survival (Glavey et al., 2014). In particular, it was shown that ST3GAL6 knock-down reduced MM adhesion on BM stromal cells and transendothelial migration in vitro and $\mathrm{MM}$ homing into the $\mathrm{BM}$ in vivo. This study revealed for the first time a functional link between sialylation and homing of $\mathrm{MM}$ into the $\mathrm{BM}$ and inspired subsequent studies aiming at understanding the underlying molecular mechanism(s). ST3GAL6 contributes to the generation of sialofucosylated structures such as $\mathrm{Sle}^{\mathrm{a} / \mathrm{x}}$ that are recognized by E-selectin (Yang et al., 2012) and may directly participate to the generation of E-selectin ligands on MM cells. Importantly, E-selectin is constitutively expressed on the BM endothelium (Schweitzer et al., 1996; Sipkins et al., 2005) and plays a crucial role in homing of human hematopoietic stem cells (HSC) into the BM (Hidalgo et al., 2002). It has also been shown that Eselectin drives cancer metastasis into the BM (Price et al., 2016; Esposito et al., 2019). Since MM metastasizes into the BM and expresses high levels of ST3GAL6, it is conceivable to hypothesize that E-selectin, together with $\mathrm{SDF} 1 \alpha$, plays an important role 
in MM homing. Indeed, it was recently shown that E-selectin ligands expressed on MM cells played a role in BM homing and possibly retention of the malignant cells in the BM (MartinezMoreno et al., 2016; Natoni et al., 2017). Notably, it was shown that MM cells enriched for E-selectin ligands recognized by

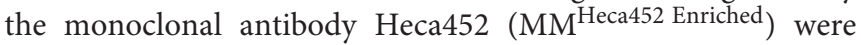
resistant to bortezomib treatment in vivo, and this resistance was reversed by a small glycomimetic molecule, GMI-1271, which inhibits E-selectin/E-selectin ligand interaction (Natoni et al., 2017). The mechanism(s) of this bortezomib resistance displayed in vivo by the $\mathrm{MM}^{\mathrm{Heca} 452}$ Enriched cells is still not known. An intriguing hypothesis is that the presence of E-selectin ligands on the $\mathrm{MM}^{\mathrm{Hec}} 452$ Enriched cells facilitate their homing into the $\mathrm{BM}$, which then provides protection to bortezomib-induced cell death. In this scenario, E-selectin ligands ensure efficient trafficking into the $\mathrm{BM}$, decreasing the time $\mathrm{MM}^{\mathrm{Heca} 452}$ Enriched cells are exposed to bortezomib in the circulation. In line with this hypothesis, we found that GMI-1271 treatment induced an accumulation of $\mathrm{MM}^{\mathrm{Heca} 452}$ Enriched cells in the peripheral blood where they may be more sensitive to bortezomib. Alternatively, E-selectin may retain the $\mathrm{MM}^{\mathrm{Heca}} 452$ Enriched cells into the BM and GMI-1271 induced egress of these cells into the circulation, a mechanism that has been proposed for leukemic stem cells (Winkler et al., 2014). Obviously, a combination of both mechanisms could be possible.

In addition to the generation of selectin ligands, sialylation plays other roles in MM. Indeed, we have recently shown that the sialylation status of the $\alpha 4$ subunit of integrins $\alpha 4 \beta 1$ and $\alpha 4 \beta 7$ modulates their activity (Natoni et al., 2019). Specifically, we have shown that inhibition of sialylation using $3 \mathrm{~F}_{\mathrm{ax}}-\mathrm{Neu} 5 \mathrm{Ac}$, a global inhibitor of the ST family (Rillahan et al., 2012), impaired maturation of the $\alpha 4$ chain, greatly reducing the ability of MM cells to interact with VCAM-1 and mucosal vascular addressin cell adhesion molecule 1 (MADCAM-1). VCAM-1, the counter-receptor for integrin $\alpha 4 \beta 1$, is expressed by the BM endothelial and stromal cells (Simmons et al., 1992; Schweitzer et al., 1996) and participates in MM adhesion on BM endothelial cells (Okada et al., 1999; Parmo-Cabanas et al., 2004). MADCAM-1, the counter-receptor for integrin $\alpha 4 \beta 7$, is also expressed in the BM and promotes homing of HSCs into the BM (Katayama et al., 2004; Tada et al., 2008; Murakami et al., 2016). Similarly, MADCAM-1 together with E-selectin and SDF1 $\alpha$ could facilitate homing of MM into the BM. Thus, global inhibition of sialylation may have a broad impact on MM homing. Indeed, we have shown that using a mouse model identical to our previous study, $3 \mathrm{~F}_{\mathrm{ax}}-\mathrm{Neu} 5 \mathrm{Ac}$ not only improved survival by enhancing bortezomib sensitivity but also prolonged survival and reduced tumor burden even in the absence of bortezomib, indicating that inhibition of sialylation was beneficial even without the addition of chemotherapy (Natoni et al., 2019).

Sialylation seems to regulate the function of CD147, also known as extracellular matrix metalloproteinase inducer (EMMPRIN). CD147 has been shown to be involved in MM proliferation and metastasis (Zhu et al., 2015). Importantly, sialylation of CD147 is upregulated by IL-6 (Wang et al., 2017). This observation opens the fascinating possibility that sialylation is also regulated by the microenvironment. Indeed, in the same study, it was shown that IL-6 transcriptionally upregulated several STs, such as ST3GAL3, ST3GAL6, and ST6GAL1 through the activation of signal transducer and activator of transcription 3 (STAT3) (Wang et al., 2017). It should be noted that ST6GAL1 has been proposed to be the main ST regulating the sialylation of the integrin subunit $\beta 1$; thus, it might impact migration, adhesion, and dissemination of MM cells (Seales et al., 2005; Christie et al., 2008; Shaikh et al., 2008).

Another microenvironmental factor that regulates the expression of STs and, in particular, ST3GAL6 is hypoxia (Glavey et al., 2014). MM develops in the BM, which represents an extremely hypoxic niche (Hu et al., 2010), and it has been shown that hypoxia promotes MM dissemination (Azab et al., 2012). Thus, upregulation of STs may represent one of the mechanisms by which hypoxia facilitates metastatic spread of MM cells.

Besides the microenvironment, ST expression in MM can also be regulated by long non-coding RNAs (lncRNAs). Indeed, it has been recently shown that the mRNA levels of ST3GAL6 are regulated by the IncRNA ST3GAL6-AS1 (Shen et al., 2018a; Vinci et al., 2018). Interestingly, ST3GAL6-AS1 is upregulated in MM, and its mRNA levels directly correlate with those of ST3GAL6 (Shen et al., 2018b; Vinci et al., 2018). Knocking down ST3GAL6AS1 results in decreased migration, invasion, and adhesion of MM to fibronectin, HUVEC, and collagen type I (Shen et al., 2018a). Although the molecular mechanisms responsible for these phenotypes are still unknown, ST3GAL6-AS1 knock-down causes a decrease in ST3GAL6 levels, suggesting co-regulatory mechanism(s), resulting in impaired sialylation.

It has been recently shown that the monoclonal immunoglobulins secreted by the malignant plasma cells display an aberrant glycosylation pattern (Zhang Z. et al., 2019). In particular, a detailed analysis of the total protein $N$-glycans derived from serum samples of MM patients showed a decrease in the $\alpha 2-3$ - and $\alpha 2-6$-linked sialic acids on secreted monoclonal immunoglobulins. Thus, it might be possible that alterations in the ST expression pattern in MM favor an increase in sialylation of the cell surface over secreted proteins, although this needs to be confirmed. Moreover, in the previous study, the $\mathrm{N}$-glycans, but not $\mathrm{O}$-glycans, were analyzed; thus, a more comprehensive view of the sialylation status of MM secreted proteins is still lacking.

As all these studies point out, aberrant sialylation plays an important role in $\mathrm{MM}$, especially in the metastatic spread of the malignant cells and in their retention into the BM niche. The relevance of sialylation in $\mathrm{MM}$ is reinforced by the observation that the expression of ST genes such as ST3GAL6 and ST3GAL1, either alone or in combination with other glycogenes, can identify patients with poor outcome (Glavey et al., 2013; Connolly et al., 2016). While ST3GAL6 has been implicated in the generation of E-selectin ligands (Yang et al., 2012), ST3GAL1 acts predominantly on Core-1 Oglycans, masking the tumor from surrounding immune cells (Burchell et al., 1999). Therefore, sialylation may well-represent an important target for future therapeutic strategies especially in combination with standard and novel chemotherapeutic drugs. However, a major obstacle to clinical development of current 
ST inhibitors is their potential to induce nephrotoxicity, which appears to be the major dose-limiting toxicity in in vivo studies (Macauley et al., 2014). This is particularly relevant in MM where renal impairment is common. While novel and more specific ST inhibitors may overcome this problem, targeted delivery systems capable of selectively releasing ST inhibitors into the BM microenvironment or the MM cells, could represent an attractive alternative solution. Besides nephrotoxicity, other potential side effects of global sialylation inhibition are those related to the hematopoietic system. Since sialic acid is an important determinant of E-, P-, and L-selectin ligands present on the immune cells (Varki, 1994; Sperandio, 2006; Zarbock et al., 2011), global sialylation inhibition will inevitably affect their biological functions, in particular leukocyte trafficking and extravasation. Elevated levels of $\alpha 2$-3-linked sialic acid together with overexpression of ST3GAL6 have been found in CD133 positive hematopoietic stem and progenitor cells (Hemmoranta et al., 2007). Moreover, it has been shown that expression of Eselectin in the $\mathrm{BM}$ is required for HSC homing into the $\mathrm{BM}$ (Hidalgo et al., 2002). Thus, sialylation inhibition would probably lead to hematopoietic stem and progenitor cell mobilization and impair their homing into the BM. However, it should be noted that E-selectin inhibition using the specific E-selectin inhibitor
GMI1271 in clinical trials in MM (NCT02811822) and AML (NCT02306291) has not been associated with myelosuppression or other side effects.

\section{LIPOSOME-BASED TARGETED DELIVERY IN MM}

Nanomedicine approaches offer the potential of enhanced and more personalized therapeutics in $\mathrm{MM}$ with a reduced risk of off-target effects (Table 1). Current treatments in MM rely on immunomodulatory drugs such as lenalidomide combined with proteasome inhibitors (PI) such as bortezomib (De La Puente and Azab, 2017). The introduction of PI inhibitors has contributed to major improvements in patient survival, but off-target effects, in particular neurotoxicity, remain a major concern (Richardson et al., 2009). Moreover, the emergence of drug resistance and how to deal with this has become a burning issue of research in MM. The tumor physiology offers a wide range of targeting opportunity, which can be designed or tuned and simultaneously co-delivered with chemotherapeutics for more personalized therapy in MM. Several groups have developed different systems to facilitate targeted delivery of chemotherapeutics in MM

TABLE 1 | Liposome-based targeted treatment available for MM.

\begin{tabular}{|c|c|c|c|c|c|}
\hline References & Nanoparticles & Drug loaded & Targeting moiety & In vitro study & In vivo study \\
\hline Ashley et al., 2014 & Liposomes & Carfilzomib & VLA-4 targeted & MM1S and $\mathrm{NCl}-\mathrm{H} 929$ cell & $\begin{array}{l}\text { SCID mice injected with } \\
\mathrm{NCl}-\mathrm{H} 929 \text { tumors }\end{array}$ \\
\hline Braham et al., 2018 & Liposomes & $\begin{array}{l}\text { Doxorubicin } \\
\text { Bortezomib }\end{array}$ & VLA-4 targeted & $\begin{array}{l}\text { BM myeloma multipotent } \\
\text { mesenchymal stromal cells, } \\
\text { endothelial progenitor cells, } \\
\text { and L363, and MM1S cells } \\
\text { co-cultured in hydrogel. }\end{array}$ & N/A \\
\hline Chang et al., 2016 & Liposomes & Paclitaxel & $\begin{array}{l}\text { Alendronate and } \\
\text { transferrin }\end{array}$ & MM1S & $\begin{array}{l}\text { MM1S GFP }{ }^{+} \text {cells were } \\
\text { injected into each mouse via } \\
\text { the tail vein to prepare the } \\
\text { tumor-bearing model }\end{array}$ \\
\hline $\begin{array}{l}\text { Lopes De Menezes et al., } \\
2000\end{array}$ & Liposomes & Doxorubicin & Anti-CD 19 & $\begin{array}{l}\text { Heterogeneous mixture of } \\
\text { PBMC from MM patients } \\
\text { and } \mathrm{ARH} 77 \text { cell }\end{array}$ & N/A \\
\hline Maillard et al., 2005 & Liposome & $\begin{array}{l}\text { Hydroxy-tamoxifen } \\
(4-H T) \text { or RU } 58668\end{array}$ & N/A & RPMI 8226 & $\begin{array}{l}\text { RPMI } 8226 \text { in female nude } \\
\text { mice }\end{array}$ \\
\hline Swami et al., 2014 & PEG PLGA & Bortezomib & Alendronate & MM1S & $\begin{array}{l}\text { NOD/SCID mice injected } \\
\text { with MM1S GFP }+ \text { Luc }^{+} \\
\text {cells }\end{array}$ \\
\hline De La Puente et al., 2018 & Chitosan & Bortezomib & anti-CD38 & $\begin{array}{l}\text { MM1S, RPMI 8226, } \\
\text { NCl-H929, and U266 }\end{array}$ & $\begin{array}{l}\text { MM1S GFP }+ \text { Luc }^{+} \text {-injected } \\
\text { SCID mice }\end{array}$ \\
\hline Yang et al., 2015 & $\mathrm{Fe}_{3} \mathrm{O}_{4}$ & Paclitaxel & $\begin{array}{l}\text { Monoclonal antibody } \\
\text { against ABCG2 }\end{array}$ & $\begin{array}{l}\text { RPMI } 8266 \text { cells and BM } \\
\text { mononuclear cells }\end{array}$ & $\begin{array}{l}\text { MM CSCs from human MM } \\
\text { RPMI } 8226 \text { cells based on } \\
\text { the CD138 }{ }^{-} \text {CD } 34^{-} \text {cell } \\
\text { phenotypes injected in } \\
\text { NOD/SCID }\end{array}$ \\
\hline Kotagiri et al., 2018 & Nano micelles & Titanocene & VLA-4 targeted & MM1S & $\begin{array}{l}\text { MM1S Luc }{ }^{+} \text {cells in SCID } \\
\text { mice }\end{array}$ \\
\hline
\end{tabular}

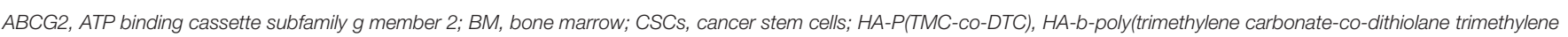

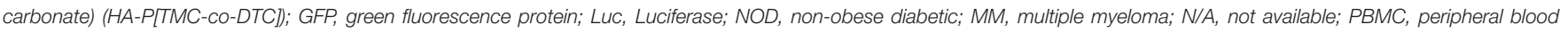
mononuclear cells; PEG, polyethylene glycol; PLGA, poly(lactic-co-glycolic acid); SCID, severe combined immunodeficiency; VLA, very late antigen. 
(Du et al., 2012). Targeting tumor physiology using antibodies, functional groups, or small molecules seems to be a more rational approach to minimize off-target effects. Kiziltepe et al. have shown that delivering doxorubicin using a lipid-PEG block polymer nano-formulation tagged by the VLA-4 antigen was significantly more cytotoxic to MM cells compared to the free drug (Kiziltepe et al., 2012). Using an alternative approach, Chang et al. developed dioleoyl phosphatidic acid (DOPA)based paclitaxel (PTX)-loaded liposomes with modifications of alendronate and transferrin, which were able to target the bone microenvironment (Chang et al., 2016). Targeting the CD38 receptor in $\mathrm{MM}$ represents an additional attractive approach as CD38 is highly expressed on malignant plasma cells (De La Puente et al., 2018). Azab's group successfully prepared a chitosan nanoparticle formulation tagged with anti-CD38 monoclonal antibodies to deliver bortezomib in vivo, improving its therapeutic efficacy and reducing side effects (De La Puente et al., 2018). This formulation showed increased therapeutic activity and reduced off-target effects compared to the free drug. Thus, targeted delivery of chemotherapeutic drugs has proven valuable in $\mathrm{MM}$, and a similar approach could be used to safely deliver $3 \mathrm{~F}_{\mathrm{ax}}-\mathrm{Neu}$ Ac, avoiding kidney toxicity. Indeed, Büll et al. have shown that encapsulation of $3 \mathrm{~F}_{\mathrm{ax}}-\mathrm{Neu} 5 \mathrm{Ac}$ in poly(lacticco-glycolic acid) (PLGA)-based nanoparticles (NPs) targeting the melanoma antigen tyrosinase related protein-1 (TRP-1) allowed specific and prolonged blockade of sialic acid expression in vitro and precluded metastatic spread in vivo (Bull et al., 2015). A similar strategy could be employed using antibodies specific to MM antigens (such as CD38 and BCMA) or by incorporating bisphosphonates into the nanoparticles to target the BM (Swami et al., 2014). Inhibiting sialylation using these approaches could have also beneficial effects on the tumor microenvironment. For instance, it has been recently reported that sialic acid blockade via intra-tumoral injection of $3 \mathrm{~F}_{\mathrm{ax}}-\mathrm{Neu} 5 \mathrm{Ac}$ could suppress tumor growth by enhancing $\mathrm{T}$ cell-mediated tumor immunity (Bull et al., 2018). Indeed, ST inhibition increased the number of activated effector immune cells, including $\mathrm{CD} 8^{+} \mathrm{T}$ cells and natural killer $(\mathrm{NK})$ cells, along with a reduction in regulatory $\mathrm{T}$ cells (Tregs) and activation of dendritic cells (DCs).

\section{REFERENCES}

Alsayed, Y., Ngo, H., Runnels, J., Leleu, X., Singha, U. K., Pitsillides, C. M., et al. (2007). Mechanisms of regulation of CXCR4/SDF-1 (CXCL12)dependent migration and homing in multiple myeloma. Blood 109, 2708-2717. doi: 10.1182/blood-2006-07-035857

Amado, M., Carneiro, F., Seixas, M., Clausen, H., and Sobrinho-Simoes, M. (1998). Dimeric sialyl-Le(x) expression in gastric carcinoma correlates with venous invasion and poor outcome. Gastroenterology 114, 462-470. doi: 10.1016/S0016-5085(98)70529-3

Ashley, J. D., Stefanick, J. F., Schroeder, V. A., Suckow, M. A., Alves, N. J., Suzuki, R., et al. (2014). Liposomal carfilzomib nanoparticles effectively target multiple myeloma cells and demonstrate enhanced efficacy in vivo. J. Control. Release 196, 113-121. doi: 10.1016/j.jconrel.2014.10.005

Azab, A. K., Hu, J., Quang, P., Azab, F., Pitsillides, C., Awwad, R., et al. (2012). Hypoxia promotes dissemination of multiple myeloma through acquisition of epithelial to mesenchymal transition-like features. Blood 119, 5782-5794. doi: 10.1182/blood-2011-09-380410

\section{CONCLUSION}

Using different systems, it should be possible to safely target the delivery of $3 \mathrm{~F}_{\mathrm{ax}}-\mathrm{Neu} 5 \mathrm{Ac}$ to the BM to inhibit sialylation in MM. We are currently working on a delivery system that employs $3 \mathrm{~F}_{\mathrm{ax}}-\mathrm{Neu} 5 \mathrm{Ac}$ encapsulated into liposomes functionalized with bisphosphonates for selective delivery into the BM with promising results. This approach has the potential to block homing of MM into the BM and mobilize the malignant cells into the circulation, making them more sensitive to chemotherapeutic drugs. Moreover, release of $3 \mathrm{~F}_{\mathrm{ax}}-\mathrm{Neu} 5 \mathrm{Ac}$ in the $\mathrm{BM}$ could have profound effects on the BM microenvironment. Indeed, we have recently shown that BM stromal cells cultured under conditions that mimic the inflammatory BM microenvironment in MM become highly immune suppressive and that this phenotype could be completely reverted by $3 \mathrm{~F}_{\mathrm{ax}}-\mathrm{Neu} 5 \mathrm{Ac}$ (Lynch et al., 2017). Thus, sialylation is a valuable target in both MM cells and the BM microenvironment.

\section{AUTHOR CONTRIBUTIONS}

AN and RB conceived and wrote the manuscript. AP and MO'D proofed and approved the final version of the manuscript.

\section{FUNDING}

This work was supported by the Health Research Board (CSA 2012/10) and Science Foundation Ireland (SFI) cofunded under the European Regional Development Programme (13/RC/2073). RB was supported by the Irish Research Council under the Government of Ireland Postdoctoral Fellowship Grant (GOIPD/2017/1283).

\section{ACKNOWLEDGMENTS}

We would like to thank all the members of MO'D and AP laboratory for their contributions and support. We apologize to all colleagues whose important work could not be cited due to space limitations.

Azab, A. K., Runnels, J. M., Pitsillides, C., Moreau, A. S., Azab, F., Leleu, X., et al. (2009). CXCR4 inhibitor AMD3100 disrupts the interaction of multiple myeloma cells with the bone marrow microenvironment and enhances their sensitivity to therapy. Blood 113, 4341-4351. doi: 10.1182/blood-2008-10-186668

Baldus, S. E., Zirbes, T. K., Monig, S. P., Engel, S., Monaca, E., Rafiqpoor, K., et al. (1998). Histopathological subtypes and prognosis of gastric cancer are correlated with the expression of mucin-associated sialylated antigens: Sialosyl-Lewis(a), Sialosyl-Lewis(x) and sialosyl-Tn. Tumour Biol. 19, 445-453. doi: 10.1159/000030036

Ben-David, T., Sagi-Assif, O., Meshel, T., Lifshitz, V., Yron, I., and Witz, I. P. (2008). The involvement of the sLe-a selectin ligand in the extravasation of human colorectal carcinoma cells. Immunol. Lett. 116, 218-224. doi: 10.1016/j.imlet.2007.11.022

Bianchi, G., and Munshi, N. C. (2015). Pathogenesis beyond the cancer clone(s) in multiple myeloma. Blood 125, 3049-3058. doi: 10.1182/blood-2014-11-568881

Braham, M. V., Deshantri, A. K., Minnema, M. C., Oner, F. C., Schiffelers, R. M., Fens, M. H., et al. (2018). Liposomal drug delivery in an in vitro 3D bone 
marrow model for multiple myeloma. Int. J. Nanomedicine 13, 8105-8118. doi: $10.2147 /$ IJN.S184262

Bull, C., Boltje, T. J., Balneger, N., Weischer, S. M., Wassink, M., Van Gemst, J. J., et al. (2018). Sialic acid blockade suppresses tumor growth by enhancing T-cell-mediated tumor immunity. Cancer Res. 78, 3574-3588. doi: 10.1158/0008-5472.CAN-17-3376

Bull, C., Boltje, T. J., Van Dinther, E. A., Peters, T., De Graaf, A. M., Leusen, J. H., et al. (2015). Targeted delivery of a sialic acid-blocking glycomimetic to cancer cells inhibits metastatic spread. ACS Nano 9, 733-745. doi: 10.1021/nn5061964

Burchell, J., Poulsom, R., Hanby, A., Whitehouse, C., Cooper, L., Clausen, H., et al. (1999). An alpha2,3 sialyltransferase (ST3Gal I) is elevated in primary breast carcinomas. Glycobiology 9, 1307-1311. doi: 10.1093/glycob/9.12.1307

Chang, Q., Geng, R., Wang, S., Qu, D., and Kong, X. (2016). DOPAbased paclitaxel-loaded liposomes with modifications of transferrin and alendronate for bone and myeloma targeting. Drug Deliv. 23, 3629-3638. doi: 10.1080/10717544.2016.1214989

Chien, S., Haq, S. U., Pawlus, M., Moon, R. T., Estey, E. H., Appelbaum, F. R., et al. (2013). Adhesion of acute myeloid leukemia blasts to E-selectin in the vascular niche enhances their survival by mechanisms such as Wnt activation. Blood 122:62.

Christie, D. R., Shaikh, F. M., Lucas, J. A. IV, Lucas, J. A. III, and Bellis, S. L. (2008). ST6Gal-I expression in ovarian cancer cells promotes an invasive phenotype by altering integrin glycosylation and function. J. Ovarian Res. 1:3. doi: 10.1186/1757-2215-1-3

Clark, E. A., and Giltiay, N. V. (2018). CD22: a regulator of innate and adaptive B cell responses and autoimmunity. Front. Immunol. 9:2235. doi: 10.3389/fimmu.2018.02235

Cluxton, C. D., Spillane, C., O'toole, S. A., Sheils, O., Gardiner, C. M., and O'leary, J. J. (2019). Suppression of natural killer cell NKG2D and CD226 antitumour cascades by platelet cloaked cancer cells: implications for the metastatic cascade. PLoS ONE 14:e0211538. doi: 10.1371/journal.pone.0211538

Cohen, A. M., Allalouf, D., Bessler, H., Djaldetti, M., Malachi, T., and Levinsky, H. (1989). Sialyltransferase activity and sialic-acid levels in multiplemyeloma and monoclonal gammopathy. Eur. J. Haematol. 42, 289-292. doi: 10.1111/j.1600-0609.1989.tb00114.x

Colombo, M., Giannandrea, D., Lesma, E., Basile, A., and Chiaramonte, R. (2019). Extracellular vesicles enhance multiple myeloma metastatic dissemination. Int. J. Mol. Sci. 20:E3236. doi: 10.3390/ijms20133236

Connolly, C., Jha, A., Natoni, A., and O'dwyer, M. E. (2016). A 13-glycosylation gene signature in multiple myeloma can predicts survival and identifies candidates for targeted therapy (GiMM13). Blood 128:4423.

Damiano, J. S., Cress, A. E., Hazlehurst, L. A., Shtil, A. A., and Dalton, W. S. (1999). Cell adhesion mediated drug resistance (CAM-DR): role of integrins and resistance to apoptosis in human myeloma cell lines. Blood 93, 1658-1667.

De La Puente, P., and Azab, A. K. (2017). Nanoparticle delivery systems, general approaches, and their implementation in multiple myeloma. Eur. J. Haematol. 98, 529-541. doi: 10.1111/ejh.12870

De La Puente, P., Luderer, M. J., Federico, C., Jin, A., Gilson, R. C., Egbulefu, C., et al. (2018). Enhancing proteasome-inhibitory activity and specificity of bortezomib by CD38 targeted nanoparticles in multiple myeloma. J. Control. Release 270, 158-176. doi: 10.1016/j.jconrel.2017.11.045

Di Marzo, L., Desantis, V., Solimando, A. G., Ruggieri, S., Annese, T., Nico, B., et al. (2016). Microenvironment drug resistance in multiple myeloma: emerging new players. Oncotarget 7, 60698-60711. doi: 10.18632/oncotarget.10849

Du, B. Y., Song, W., Bai, L., Shen, Y., Miao, S. Y., and Wang, L. F. (2012). Synergistic effects of combination treatment with bortezomib and doxorubicin in human neuroblastoma cell lines. Chemotherapy 58, 44-51. doi: $10.1159 / 000335603$

Esposito, M., Mondal, N., Greco, T. M., Wei, Y., Spadazzi, C., Lin, S. C., et al. (2019). Bone vascular niche E-selectin induces mesenchymalepithelial transition and Wnt activation in cancer cells to promote bone metastasis. Nat. Cell Biol. 21, 627-639. doi: 10.1038/s41556-0190309-2

Fedarko, N. S., Fohr, B., Robey, P. G., Young, M. F., and Fisher, L. W. (2000). Factor $\mathrm{H}$ binding to bone sialoprotein and osteopontin enables tumor cell evasion of complement-mediated attack. J. Biol. Chem. 275, 16666-16672. doi: 10.1074/jbc.M001123200
Florena, A. M., Tripodo, C., Miceli, L., Ingrao, S., Porcasi, R., and Franco, V. (2005). Identification of CD162 in plasma-cell dyscrasia. Lancet Oncol. 6:632. doi: 10.1016/S1470-2045(05)70287-4

Fukuoka, K., Narita, N., and Saijo, N. (1998). Increased expression of sialyl Lewis(x) antigen is associated with distant metastasis in lung cancer patients: immunohistochemical study on bronchofiberscopic biopsy specimens. Lung Cancer 20, 109-116. doi: 10.1016/S0169-5002(98)00016-6

Gakhar, G., Navarro, V. N., Jurish, M., Lee, G. Y., Tagawa, S. T., Akhtar, N. H., et al. (2013). Circulating tumor cells from prostate cancer patients interact with E-selectin under physiologic blood flow. PLoS ONE 8:e85143. doi: 10.1371/journal.pone.0085143

Garnham, R., Scott, E., Livermore, K. E., and Munkley, J. (2019). ST6GAL1: a key player in cancer. Oncol. Lett. 18, 983-989. doi: 10.3892/ol.2019.10458

Gazitt, Y., and Akay, C. (2004). Mobilization of myeloma cells involves SDF1/CXCR4 signaling and downregulation of VLA-4. Stem Cells 22, 65-73. doi: 10.1634/stemcells.22-1-65

Gelberg, H., Healy, L., Whiteley, H., Miller, L. A., and Vimr, E. (1996). In vivo enzymatic removal of alpha 2->6-linked sialic acid from the glomerular filtration barrier results in podocyte charge alteration and glomerular injury. Lab. Invest. 74, 907-920.

Geng, Y., Yeh, K., Takatani, T., and King, M. R. (2012). Three to tango: MUC1 as a ligand for both E-selectin and ICAM-1 in the breast cancer metastatic cascade. Front. Oncol. 2:76. doi: 10.3389/fonc.2012.00076

Glavey, S., Wu, P., Murillo, L. S., Loughrey, C., Roccaro, A. M., Morgan, G. J., et al. (2013). Low expression of the FUCA1 gene is an adverse prognostic factor in myeloma and combined with high sialyltransferase gene expression identifies patients at increased risk of early disease progression and death. Blood 122:1864.

Glavey, S. V., Manier, S., Natoni, A., Sacco, A., Moschetta, M., Reagan, M. R., et al. (2014). The sialyltransferase ST3GAL6 influences homing and survival in multiple myeloma. Blood 124, 1765-1776. doi: 10.1182/blood-2014-03-560862

Gout, S., Tremblay, P. L., and Huot, J. (2008). Selectins and selectin ligands in extravasation of cancer cells and organ selectivity of metastasis. Clin. Exp. Metastasis 25, 335-344. doi: 10.1007/s10585-007-9096-4

Grabowski, P., Mann, B., Mansmann, U., Lovin, N., Foss, H. D., Berger, G., et al. (2000). Expression of SIALYL-Le(x) antigen defined by MAb AM-3 is an independent prognostic marker in colorectal carcinoma patients. Int. J. Cancer 88, 281-286. doi: 10.1002/1097-0215(20001015)88:2<281::AID-IJC21>3.0.CO;2-2

Harduin-Lepers, A., Vallejo-Ruiz, V., Krzewinski-Recchi, M. A., Samyn-Petit, B., Julien, S., and Delannoy, P. (2001). The human sialyltransferase family. Biochimie 83, 727-737. doi: 10.1016/S0300-9084(01)01301-3

Hemmoranta, H., Satomaa, T., Blomqvist, M., Heiskanen, A., Aitio, O., Saarinen, J., et al. (2007). N-glycan structures and associated gene expression reflect the characteristic N-glycosylation pattern of human hematopoietic stem and progenitor cells. Exp. Hematol. 35, 1279-1292. doi: 10.1016/j.exphem.2007.05.006

Hidalgo, A., Weiss, L. A., and Frenette, P. S. (2002). Functional selectin ligands mediating human CD34 $(+)$ cell interactions with bone marrow endothelium are enhanced postnatally. J. Clin. Invest. 110, 559-569. doi: 10.1172/JCI0 214047

Hu, J., Handisides, D. R., Van Valckenborgh, E., De Raeve, H., Menu, E., Vande Broek, I., et al. (2010). Targeting the multiple myeloma hypoxic niche with TH-302, a hypoxia-activated prodrug. Blood 116, 1524-1527. doi: 10.1182/blood-2010-02-269126

Katayama, Y., Hidalgo, A., Peired, A., and Frenette, P. S. (2004). Integrin alpha4beta7 and its counterreceptor MAdCAM-1 contribute to hematopoietic progenitor recruitment into bone marrow following transplantation. Blood 104, 2020-2026. doi: 10.1182/blood-2003-12-4157

Kiziltepe, T., Ashley, J. D., Stefanick, J. F., Qi, Y. M., Alves, N. J., Handlogten, M. W., et al. (2012). Rationally engineered nanoparticles target multiple myeloma cells, overcome cell-adhesion-mediated drug resistance, and show enhanced efficacy in vivo. Blood Cancer J. 2:e64. doi: 10.1038/bcj.2012.10

Kotagiri, N., Cooper, M. L., Rettig, M., Egbulefu, C., Prior, J., Cui, G., et al. (2018). Radionuclides transform chemotherapeutics into phototherapeutics for precise treatment of disseminated cancer. Nat. Commun. 9, 275-286. doi: 10.1038/s41467-017-02758-9 
Labelle, M., Begum, S., and Hynes, R. O. (2014). Platelets guide the formation of early metastatic niches. Proc. Natl. Acad. Sci. U.S.A. 111, E3053-3061. doi: $10.1073 /$ pnas. 1411082111

Li, B., Hong, J., Hong, M., Wang, Y., Yu, T., Zang, S., et al. (2019). piRNA-823 delivered by multiple myeloma-derived extracellular vesicles promoted tumorigenesis through re-educating endothelial cells in the tumor environment. Oncogene 38, 5227-5238. doi: 10.1038/s41388-019-0788-4

Li, J., Guillebon, A. D., Hsu, J. W., Barthel, S. R., Dimitroff, C. J., Lee, Y. F., et al. (2013). Human fucosyltransferase 6 enables prostate cancer metastasis to bone. Br. J. Cancer 109, 3014-3022. doi: 10.1038/bjc.2013.690

Liu, Y., Zhu, X. J., Zeng, C., Wu, P. H., Wang, H. X., Chen, Z. C., et al. (2014). Microvesicles secreted from human multiple myeloma cells promote angiogenesis. Acta Pharmacol. Sin. 35, 230-238. doi: 10.1038/aps.2013.141

Lopes De Menezes, D. E., Pilarski, L. M., Belch, A. R., and Allen, T. M. (2000). Selective targeting of immunoliposomal doxorubicin against human multiple myeloma in vitro and ex vivo. Biochim. Biophys. Acta 1466, 205-220. doi: 10.1016/S0005-2736(00)00203-0

Lynch, K., Grace, O. M., Ryan, A., Ritter, T., and O’dwyer, M. (2017). Mesenchymal stromal cell sialylation enhances immune suppression in multiple myeloma. Blood 130:124.

Macauley, M. S., Arlian, B. M., Rillahan, C. D., Pang, P. C., Bortell, N., Marcondes, M. C., et al. (2014). Systemic blockade of sialylation in mice with a global inhibitor of sialyltransferases. J. Biol. Chem. 289, 35149-35158. doi: 10.1074/jbc.M114.606517

Maillard, S., Ameller, T., Gauduchon, J., Gougelet, A., Gouilleux, F., Legrand, P., et al. (2005). Innovative drug delivery nanosystems improve the antitumor activity in vitro and in vivo of anti-estrogens in human breast cancer and multiple myeloma. J. Steroid Biochem. Mol. Biol. 94, 111-121. doi: $10.1016 /$ j.jsbmb.2004.12.023

Manier, S., Sacco, A., Leleu, X., Ghobrial, I. M., and Roccaro, A. M. (2012). Bone marrow microenvironment in multiple myeloma progression. J. Biomed. Biotechnol. 2012, 157496-157500. doi: 10.1155/2012/157496

Martinez-Moreno, M., Leiva, M., Aguilera-Montilla, N., Sevilla-Movilla, S., Isern De Val, S., Arellano-Sanchez, N., et al. (2016). In vivo adhesion of malignant B cells to bone marrow microvasculature is regulated by $\alpha 4 \beta 1$ cytoplasmic-binding proteins. Leukemia $30,861-872$. doi: 10.1038/leu. 2015.332

Mcever, R. P. (2015). Selectins: initiators of leucocyte adhesion and signalling at the vascular wall. Cardiovasc. Res. 107, 331-339. doi: 10.1093/cvr/cvv154

Menu, E., Asosingh, K., Indraccolo, S., De Raeve, H., Van Riet, I., Van Valckenborgh, E., et al. (2006). The involvement of stromal derived factor lalpha in homing and progression of multiple myeloma in the 5TMM model. Haematologica 91, 605-612.

Moschetta, M., Kawano, Y., Sacco, A., Belotti, A., Ribolla, R., Chiarini, M., et al. (2017). Bone marrow stroma and vascular contributions to myeloma bone homing. Curr. Osteoporos. Rep. 15, 499-506. doi: 10.1007/s11914-017-0399-3

Murakami, J. L., Xu, B., Franco, C. B., Hu, X., Galli, S. J., Weissman, I. L., et al. (2016). Evidence that $\beta 7$ integrin regulates hematopoietic stem cell homing and engraftment through interaction with MAdCAM-1. Stem Cells Dev. 25, 18-26. doi: $10.1089 / \mathrm{scd} .2014 .0551$

Muz, B., Azab, F., De La Puente, P., Rollins, S., Alvarez, R., Kawar, Z., et al. (2015). Inhibition of P-selectin and PSGL-1 using humanized monoclonal antibodies increases the sensitivity of multiple myeloma cells to bortezomib. Biomed Res. Int. 2015, 417586-417593. doi: 10.1155/2015/417586

Natoni, A., Farrell, M. L., Harris, S., Falank, C., Kirkham-Mccarthy, L., Macauley, M. S., et al. (2019). Sialyltransferase inhibition leads to inhibition of tumor cell interactions with E-selectin, VCAM1, and MADCAM1, and improves survival in a human multiple myeloma mouse model. Haematologica. haematol.2018.212266. doi: 10.3324/haematol.2018.212266. [Epub ahead of print].

Natoni, A., Macauley, M. S., and O'dwyer, M. E. (2016). Targeting selectins and their ligands in cancer. Front. Oncol. 6:93. doi: 10.3389/fonc.2016.00093

Natoni, A., Smith, T. A. G., Keane, N., Mcellistrim, C., Connolly, C., Jha, A., et al. (2017). E-selectin ligands recognised by HECA452 induce drug resistance in myeloma, which is overcome by the E-selectin antagonist, GMI-1271. Leukemia 31, 2642-2651. doi: 10.1038/leu.2017.123

Neri, P., Ren, L., Azab, A. K., Brentnall, M., Gratton, K., Klimowicz, A. C., et al. (2011). Integrin $\beta 7$-mediated regulation of multiple myeloma cell adhesion, migration, and invasion. Blood 117, 6202-6213. doi: 10.1182/blood-2010-06-292243

Nielsen, T., Kristensen, S. R., Gregersen, H., Teodorescu, E. M., Christiansen, G., and Pedersen, S. (2019). Extracellular vesicle-associated procoagulant phospholipid and tissue factor activity in multiple myeloma. PLOS ONE 14:e0210835. doi: 10.1371/journal.pone.0210835

Okada, T., Hawley, R. G., Kodaka, M., and Okuno, H. (1999). Significance of VLA-4-VCAM-1 interaction and CD44 for transendothelial invasion in a bone marrow metastatic myeloma model. Clin. Exp. Metastasis 17, 623-629. doi: 10.1023/A:1006715504719

Parmo-Cabanas, M., Bartolome, R. A., Wright, N., Hidalgo, A., Drager, A. M., and Teixido, J. (2004). Integrin alpha4betal involvement in stromal cellderived factor-1alpha-promoted myeloma cell transendothelial migration and adhesion: role of cAMP and the actin cytoskeleton in adhesion. Exp. Cell Res. 294, 571-580. doi: 10.1016/j.yexcr.2003.12.003

Placke, T., Orgel, M., Schaller, M., Jung, G., Rammensee, H. G., Kopp, H. G., et al. (2012). Platelet-derived MHC class I confers a pseudonormal phenotype to cancer cells that subverts the antitumor reactivity of natural killer immune cells. Cancer Res. 72, 440-448. doi: 10.1158/0008-5472.CAN-11-1872

Price, T. T., Burness, M. L., Sivan, A., Warner, M. J., Cheng, R., Lee, C. H., et al. (2016). Dormant breast cancer micrometastases reside in specific bone marrow niches that regulate their transit to and from bone. Sci. Transl. Med. 8:340ra373. doi: $10.1126 /$ scitranslmed.aad4059

Remiker, A. S., and Palumbo, J. S. (2018). Mechanisms coupling thrombin to metastasis and tumorigenesis. Thromb. Res. 164(Suppl. 1), S29-S33. doi: 10.1016/j.thromres.2017.12.020

Richardson, P. G., Xie, W., Mitsiades, C., Chanan-Khan, A. A., Lonial, S., Hassoun, H., et al. (2009). Single-agent bortezomib in previously untreated multiple myeloma: efficacy, characterization of peripheral neuropathy, and molecular correlations with response and neuropathy. J. Clin. Oncol. 27, 3518-3525. doi: 10.1200/JCO.2008.18.3087

Rillahan, C. D., Antonopoulos, A., Lefort, C. T., Sonon, R., Azadi, P., Ley, K., et al. (2012). Global metabolic inhibitors of sialyl- and fucosyltransferases remodel the glycome. Nat. Chem. Biol. 8, 661-668. doi: 10.1038/nchembio.999

Rodrigues, E., and Macauley, M. S. (2018). Hypersialylation in cancer: modulation of inflammation and therapeutic opportunities. Cancers 10:E207. doi: $10.3390 /$ cancers 10060207

Rutishauser, U. (2008). Polysialic acid in the plasticity of the developing and adult vertebrate nervous system. Nat. Rev. Neurosci. 9, 26-35. doi: 10.1038/nrn2285

Sanz-Rodriguez, F., Ruiz-Velasco, N., Pascual-Salcedo, D., and Teixido, J. (1999). Characterization of VLA-4-dependent myeloma cell adhesion to fibronectin and VCAM-1. Br. J. Haematol. 107, 825-834. doi: 10.1046/j.1365-2141.1999.01762.x

Schultz, M. J., Swindall, A. F., and Bellis, S. L. (2012). Regulation of the metastatic cell phenotype by sialylated glycans. Cancer Metastasis Rev. 31, 501-518. doi: 10.1007/s10555-012-9359-7

Schweitzer, K. M., Drager, A. M., Van Der Valk, P., Thijsen, S. F., Zevenbergen, A., Theijsmeijer, A. P., et al. (1996). Constitutive expression of E-selectin and vascular cell adhesion molecule-1 on endothelial cells of hematopoietic tissues. Am. J. Pathol. 148, 165-175.

Seales, E. C., Jurado, G. A., Brunson, B. A., Wakefield, J. K., Frost, A. R., and Bellis, S. L. (2005). Hypersialylation of betal integrins, observed in colon adenocarcinoma, may contribute to cancer progression by up-regulating cell motility. Cancer Res. 65, 4645-4652. doi: 10.1158/0008-5472.CAN-04-3117

Shaikh, F. M., Seales, E. C., Clem, W. C., Hennessy, K. M., Zhuo, Y., and Bellis, S. L. (2008). Tumor cell migration and invasion are regulated by expression of variant integrin glycoforms. Exp. Cell Res. 314, 2941-2950. doi: 10.1016/j.yexcr.2008.07.021

Shen, Y., Feng, Y., Chen, H., Jia, Y., Peng, Y., Zhang, R., et al. (2018a). Silencing long non-coding rna st3gal6-as1 inhibits adhesion and migration of myeloma cells in vitro. Blood 132, 4470-4470. doi: 10.1182/blood-2018-99-114660

Shen, Y., Feng, Y. D., Chen, H. L., Huang, L. J., Wang, F. X., Bai, J., et al. (2018b). Focusing on long non-coding RNA dysregulation in newly diagnosed multiple myeloma. Life Sci. 196, 133-142. doi: 10.1016/j.lff.2018.01.025

Simmons, P. J., Masinovsky, B., Longenecker, B. M., Berenson, R., Torok-Storb, B., and Gallatin, W. M. (1992). Vascular cell adhesion molecule-1 expressed by bone marrow stromal cells mediates the binding of hematopoietic progenitor cells. Blood 80, 388-395. 
Sipkins, D. A., Wei, X., Wu, J. W., Runnels, J. M., Cote, D., Means, T. K., et al. (2005). In vivo imaging of specialized bone marrow endothelial microdomains for tumour engraftment. Nature 435, 969-973. doi: 10.1038/nature03703

Sperandio, M. (2006). Selectins and glycosyltransferases in leukocyte rolling in vivo. FEBS J. 273, 4377-4389. doi: 10.1111/j.1742-4658.2006.05437.x

Swami, A., Reagan, M. R., Basto, P., Mishima, Y., Kamaly, N., Glavey, S., et al. (2014). Engineered nanomedicine for myeloma and bone microenvironment targeting. Proc. Natl. Acad. Sci. U.S.A. 111, 10287-10292. doi: 10.1073/pnas.1401337111

Tada, T., Inoue, N., Widayati, D. T., and Fukuta, K. (2008). Role of MAdCAM-1 and its ligand on the homing of transplanted hematopoietic cells in irradiated mice. Exp. Anim. 57, 347-356. doi: 10.1538/expanim.57.347

Tatsumi, M., Watanabe, A., Sawada, H., Yamada, Y., Shino, Y., and Nakano, H. (1998). Immunohistochemical expression of the sialyl Lewis $\mathrm{x}$ antigen on gastric cancer cells correlates with the presence of liver metastasis. Clin. Exp. Metastasis 16, 743-750. doi: 10.1023/A:1006584829246

Varki, A. (1994). Selectin ligands. Proc. Natl. Acad. Sci. U.S.A. 91, 7390-7397. doi: $10.1073 /$ pnas. 91.16 .7390

Varki, A. (2008). Sialic acids in human health and disease. Trends Mol. Med. 14, 351-360. doi: 10.1016/j.molmed.2008.06.002

Vinci, C., Taiana, E., Favasuli, V., Ronchetti, D., Agnelli, L., Manzoni, M., et al. (2018). The long non-coding rna st3gal6-as1 deregulated in multiple myeloma. Haematologica 103, S23-S23.

Waldschmidt, J. M., Simon, A., Wider, D., Muller, S. J., Follo, M., Ihorst, G., et al. (2017). CXCL12 and CXCR7 are relevant targets to reverse cell adhesionmediated drug resistance in multiple myeloma. Br. J. Haematol. 179, 36-49. doi: $10.1111 /$ bjh. 14807

Wang, J., De Veirman, K., Faict, S., Frassanito, M. A., Ribatti, D., Vacca, A., et al. (2016). Multiple myeloma exosomes establish a favourable bone marrow microenvironment with enhanced angiogenesis and immunosuppression. $J$. Pathol. 239, 162-173. doi: 10.1002/path.4712

Wang, Y. M., Fan, R., Lei, L., Wang, A. Y., Wang, X. M., Ying, S., et al. (2017). Interleukin- 6 drives multiple myeloma progression through upregulating of CD147/emmprin expression and its sialylation. Blood 130:3037.

Winkler, I. G., Barbier, V., Pattabiraman, D. R., Gonda, T. J., Magnani, J. L., and Levesque, J. P. (2014). Vascular niche E-selectin protects acute myeloid leukaemia stem cells from chemotherapy. Blood 124, 620 .

Woodman, N., Pinder, S. E., Tajadura, V., Le Bourhis, X., Gillett, C., Delannoy, P., et al. (2016). Two E-selectin ligands, BST-2 and LGALS3BP, predict metastasis and poor survival of ER-negative breast cancer. Int. J. Oncol. 49, 265-275. doi: 10.3892/ijo.2016.3521
Yang, C., Xiong, F., Dou, J., Xue, J., Zhan, X., Shi, F., et al. (2015). Target therapy of multiple myeloma by PTX-NPs and ABCG2 antibody in a mouse xenograft model. Oncotarget 6, 27714-27724. doi: 10.18632/oncotarget.4663

Yang, W. H., Nussbaum, C., Grewal, P. K., Marth, J. D., and Sperandio, M. (2012). Coordinated roles of ST3Gal-VI and ST3Gal-IV sialyltransferases in the synthesis of selectin ligands. Blood 120, 1015-1026. doi: 10.1182/blood-2012-04-424366

Zarbock, A., Ley, K., Mcever, R. P., and Hidalgo, A. (2011). Leukocyte ligands for endothelial selectins: specialized glycoconjugates that mediate rolling and signaling under flow. Blood 118, 6743-6751. doi: 10.1182/blood-2011-07-343566

Zarfati, M., Avivi, I., Brenner, B., Katz, T., and Aharon, A. (2019). Extracellular vesicles of multiple myeloma cells utilize the proteasome inhibitor mechanism to moderate endothelial angiogenesis. Angiogenesis 22, 185-196. doi: 10.1007/s10456-018-9649-y

Zhang, L., Lei, Q., Wang, H., Xu, C., Liu, T., Kong, F., et al. (2019). Tumorderived extracellular vesicles inhibit osteogenesis and exacerbate myeloma bone disease. Theranostics 9, 196-209. doi: 10.7150/thno.27550

Zhang, Z., Westhrin, M., Bondt, A., Wuhrer, M., Standal, T., and Holst, S. (2019). Serum protein $\mathrm{N}$-glycosylation changes in multiple myeloma. Biochim. Biophys. Acta Gen. Subj. 1863, 960-970. doi: 10.1016/j.bbagen.2019. 03.001

Zhu, D., Wang, Z., Zhao, J. J., Calimeri, T., Meng, J., Hideshima, T., et al. (2015). The cyclophilin A-CD147 complex promotes the proliferation and homing of multiple myeloma cells. Nat. Med. 21, 572-580. doi: 10.1038/ nm.3867

Conflict of Interest: MO'D is listed as an inventor on the patent application US20170327899A1 https://patents.google.com/patent/US20170327899A1/en.

The remaining authors declare that the research was conducted in the absence of any commercial or financial relationships that could be construed as a potential conflict of interest.

Copyright (๑) 2019 Natoni, Bohara, Pandit and O'Dwyer. This is an open-access article distributed under the terms of the Creative Commons Attribution License (CC $B Y)$. The use, distribution or reproduction in other forums is permitted, provided the original author(s) and the copyright owner(s) are credited and that the original publication in this journal is cited, in accordance with accepted academic practice. No use, distribution or reproduction is permitted which does not comply with these terms. 